\title{
Semi-automated biobank sample processing with a 384 high density sample tube robot used in cancer and cardiovascular studies
}

\author{
Johan Malm ${ }^{1,2^{*}}$, Henrik Lindberg ${ }^{3}$, David Erlinge ${ }^{4}$, Roger Appelqvist ${ }^{3}$, Maria Yakovleva ${ }^{2}$, Charlotte Welinder ${ }^{2,3}$, \\ Erik Steinfelder ${ }^{5}$, Thomas E Fehniger ${ }^{2,3}$ and György Marko-Varga ${ }^{2,3,6}$
}

\begin{abstract}
Background: In the postgenomic era, it has become evident that analysis of genetic and protein expression changes alone is not sufficient to understand most disease processes in e.g. cardiovascular and cancer disease. Biobanking has been identified as an important area for development and discovery of better diagnostic tools and new treatment modalities. Biobanks are developed in order to integrate the collection of clinical samples from both healthy individuals and patients and provide valuable information that will make possible improved patient care. Modern healthcare developments are intimately linked to information based on studies of patient samples from biobank archives in large scale studies. Today biobanks form important national, as well as international, networks that share and combine global resources.
\end{abstract}

Methods: We have developed and validated a novel biobanking workflow process that utilizes 384-tube systems with a high speed sample array robot with unique processing principles.

Results: The 384-tube format and robotic processing is incorporated into a cancer and cardiovascular diagnostic/ prognostic research program with therapeutic interventions. Our biobank practice has gained acceptance within many hospitals and research units and is based on high-density sample storage with small aliquot sample volumes. The previous standard of 5-10 mL sample volume tubes is being replaced by smaller volumes of 50-70 $\mu \mathrm{L}$ blood fractions that typically result in hundreds of thousands of aliquot fractions in 384-tube systems.

Conclusions: Our novel biobanking workflow process is robust and well suited for clinical studies.

Keywords: Biobank, 384 sample tubes, Cancer, Cardiovascular

\section{Background}

Patient and society's demand and expectations in health care combined with rising costs pose a great challenge on our health care system. The scientific community is expected to develop solutions that can improve clinical outcome and increase cost efficiency without jeopardizing the quality of care of individual patients. Future health care will be tailored to the individual and the introduction of personalized medicine in routine healthcare relies

\footnotetext{
*Correspondence: johan.malm@med.lu.se

1 Section for Clinical Chemistry, Department of Translational Medicine, Lund University, Skåne University Hospital Malmö, 20502 Malmö, Sweden Full list of author information is available at the end of the article
}

heavily on large scale biorepositories, biobanks (http:// www.informatics-review.com/wiki/index.php/Biobanking_Definition) $[1,2]$.

Millions of clinical samples are collected every day for use in diagnostic tests that support clinical decision making. The majority of clinical routine samples are discarded but it is estimated that over one billion clinical samples are stored in biobanks worldwide [3]. The preservation of these samples is an important undertaking since each sample is unique and has the potential to facilitate future research and diagnostics.

Most errors in a hospital laboratory are due to preanalytical factors, if the preanalytical procedure fails subsequent downstream measurements will also fail $[4,5]$.

\section{Springer}


Preanalytical errors still account for $60-70 \%$ of all mistakes in laboratory diagnostics and in nearly one-fifth of these, errors might be associated with further inappropriate investigations and unjustifiable increase in costs [6].

The stability of a biospecimen sample depends on the method of storage. The vials must be sealed tightly, stored at the right temperature and the issue of possible freeze/thaw damage studied [7]. In almost all biobanks the samples are stored in 96-tube systems and each tube regularly used on more than one occasion. The current methods of sample handling and storage are often different in different laboratories, a fact that further complicates inter-laboratory studies, e.g. clinical multi-center studies.

Personalized medicine will be based on different diagnostic technologies used for studies of e.g. the human proteome. Results from proteome studies approaches [811] are important complements to genomic data [11-15] and provide crucial information about the target driver molecules and their post-translational modifications.

Quantitative mass spectrometry is an excellent way to identify biomarkers that can be used in personalized medicine. The technology is very sensitive and thus the sample quality is of utmost importance. We believe that new strategies must be advanced and established for the integration and use of biobank samples in routine health care, samples from patients that can be collected, processed and stored by a large scale archiving strategy.

We have previously reported on automated processing with robotic liquid handling for blood sample standardization developments for large scale biobanking $[16,17]$, as well as developments within standardized biobank workflows with evidence of sample history, tracking information, ensuring the sample integrity [18].

We hereby present a semi-automated workflow for biobank samples utilizing the high density 384-tube system where the rigidity and performance of the automated platform is used within cancer and cardiovascular studies outlined in Fig. 1.

\section{Methods}

\section{Liquid chromatography mass spectrometry}

The peptide separation was carried out using a Thermo UPLC system (EASY nLC 1000, Thermo Scientific, San José, CA, USA). Aliquots of $0.5-1 \mu \mathrm{g}$ of digested samples were injected onto a trap column (Acclaim PepMap100, C18, $5 \mu \mathrm{m}, 100 \AA, 300 \mu \mathrm{m}$ i.d. $\times 5 \mathrm{~mm}, \mathrm{PN} 160454$, Thermo Scientific, San José, CA, USA). Peptides were then separated on an analytical column (Acclaim PepMap RSLC C18, $2 \mu \mathrm{m}, 100 \AA ̊$, $75 \mu \mathrm{m}$ i.d. $\times 25 \mathrm{~cm}$, nanoViper column, PN 164536, Thermo Scientific, San José, CA, USA) using a solvent gradient $(0-40 \%$ acetonitrile in $90 \mathrm{~min}$ ). Peptides separated on nanoLC system were analyzed with Q Exactive mass spectrometer is a positive ion mode. Samples were analyzed using an spray voltage and heated capillary temperature of $1.75 \mathrm{kV}$ and $280^{\circ} \mathrm{C}$, respectively. Data was acquired in a top ten data dependent acquisition mode (DDA), in which a high resolution 70,000 (at $m / z 200)$ MS survey scan $(m / z 350-1800$, AGC value of $1 \mathrm{e} 6$, maximum injection time of $100 \mathrm{~ms}$ ) was followed by an acquisition of tandem mass spectra (35,000 (at $\mathrm{m} / \mathrm{z} 200)$, target AGC value of 1e6, maximum injection time of $120 \mathrm{~ms}$ ). For the ten most intense ions, HCD fragmentation was performed and the MS/ MS spectra were acquired. Dynamic exclusion was set to $20 \mathrm{~s}$. The proteotypic peptides for each protein as well as the best transitions for each peptide are summarized in supplement.

\section{Sample array robot}

The Sample Array Tube Handler (Thermo Scientific, San José, CA, USA) is specifically designed to be used with 384-tube plates. The platform is designed to hold up to 20 plates and can operate at lower temperatures $\left(-20^{\circ} \mathrm{C}\right)$.

Tubes can be automatically transferred from a source plate (A) to a target plate (B) using two columns Excel file containing lists of tubes with source and destination positions. There are no requirements where to place the racks, all of the positions are open for both source and target plates.

The test was performed under three different conditions:

- At room temperature.

- At $5-8^{\circ} \mathrm{C}$ (cold room).

- At $-20^{\circ} \mathrm{C}$ (freezer).

A large number of movements were made during the test period of the Sample Array robot.

We used several different picking profiles to check robot performance under all three conditions. When running the Sample Array robot in a freezer a web camera was installed together with a web interface to control the robot from outside.

\section{Results and discussion}

\section{Preserving the integrity of clinical samples}

Biobank sample integrity is paramount for subsequent studies but also other factors are important for showing the community that we are acting responsibly. Today's clinical scientist needs to be prepared to not only collect and validate their raw gene-and protein- data and to safe keep the stored samples, but also to establish and maintain a legal administration for documenting the position of every individual sample in the biobank by a process 


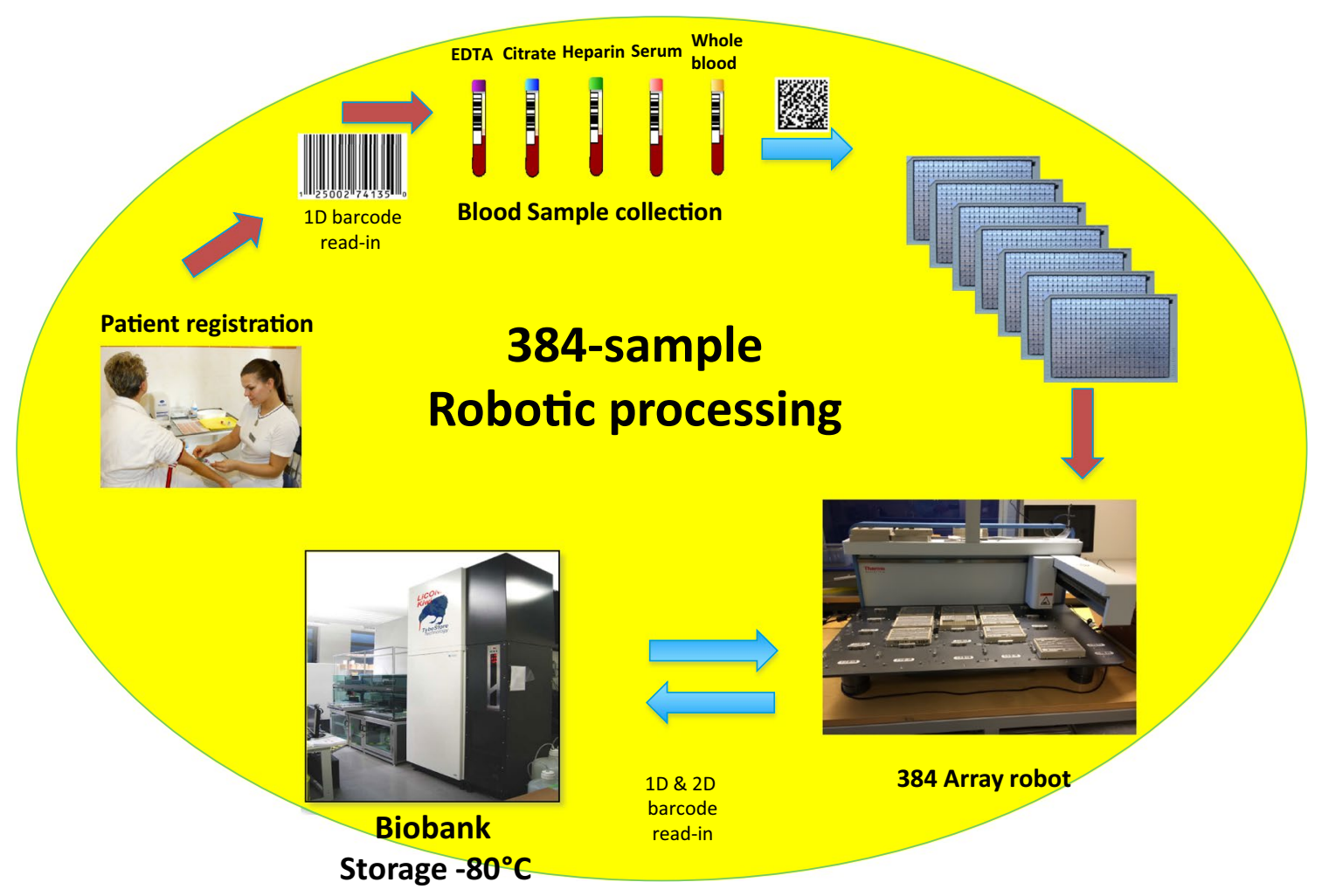

Fig. 1 Biobanking work flow in the hospital where automated processing of 384-tube systems are processed by the "384 Sample Array Robot".

that de-identifies the actual personal information linked to a specific sample. Currently the sample processing of blood samples from patients is performed at short cycle times within hospitals, typically $2 \mathrm{~h} \mathrm{[12-15].} \mathrm{The} 2 \mathrm{~h}$ include the collection from the patient, sample handling, centrifugations, and aliquoting into 384 tube racks, i.e., from the time the needle goes into the arm until the aliquoted blood fractions are stored at $-80^{\circ} \mathrm{C}$. The workflow of the semi-automated 384-tube system processing and storage is depicted in Fig. 1. The protocol is used in cancer- and cardiovascular studies. In this workflow system, each 384 rack will hold one or two patients, depending on the number of primary tubes collected in the study. Here we typically use five primary tubes $(6 \mathrm{~mL})$ with blood containing various anticoagulants (EDTA, citrate, heparin). All blood fractions end up in the same rack due to the lack of time to distribute the aliquots into multiple 384-racks. This is the price that has to be paid, in order to manage handling blood samples from patients in a time cycle of less than $2 \mathrm{~h}$.

Prior to protein expression analysis, the processing protocols are similar for both tissues from resected tumors from cancer patients, and blood samples from cardiovascular patients, as shown in Fig. 2. We have processed several series of sample cohorts in the sample work up protocols as outlined with high reproducibility and adaptability $[10-12,14]$.

Proteomics analysis of biobank samples are currently performed in a large number of studies where the output with the latest high-resolution mass spectrometers is approx 100-150,000 protein sequences for a given tumor tissue sample and approx. 10-15,000 protein sequences analysed in blood fractions such as plasma or serum. Two examples are provided where protein sequence expressions are presented for two patients (see Fig. 3).

Most of the peptides are eluted during a cycle time of $40 \mathrm{~min}$, if dead volumes are corrected for (30-70 min. These high resolution nano-chromatograms show extreme power, where the interface to the mass spectrometer is carrying out the second part of the separation along with the peptide annotation (nano LC-MS), followed by the peptide sequencing (nano LC-MS/MS).

This biobank workflow is currently utilized at the center of excellence in biological and medical mass spectrometry "CEBMMS" at Lund University in Sweden (http://cebmms.lu.se). The center is an investigator initiated activity with members in the Faculties of Medicine, Science, and Engineering, performing a number of clinical studies in collaboration with the Lund University hospitals. 


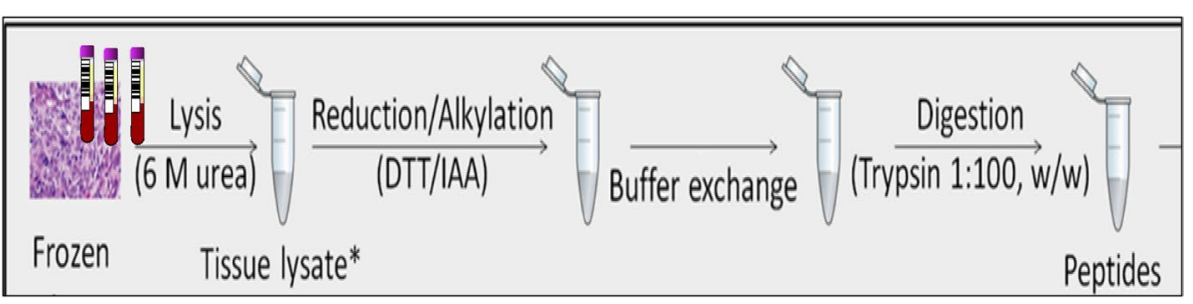

Fig. 2 Protocol used for the sample preparation of clinical patient samples within biobank studies, using both tumor tissue and blood fractions.
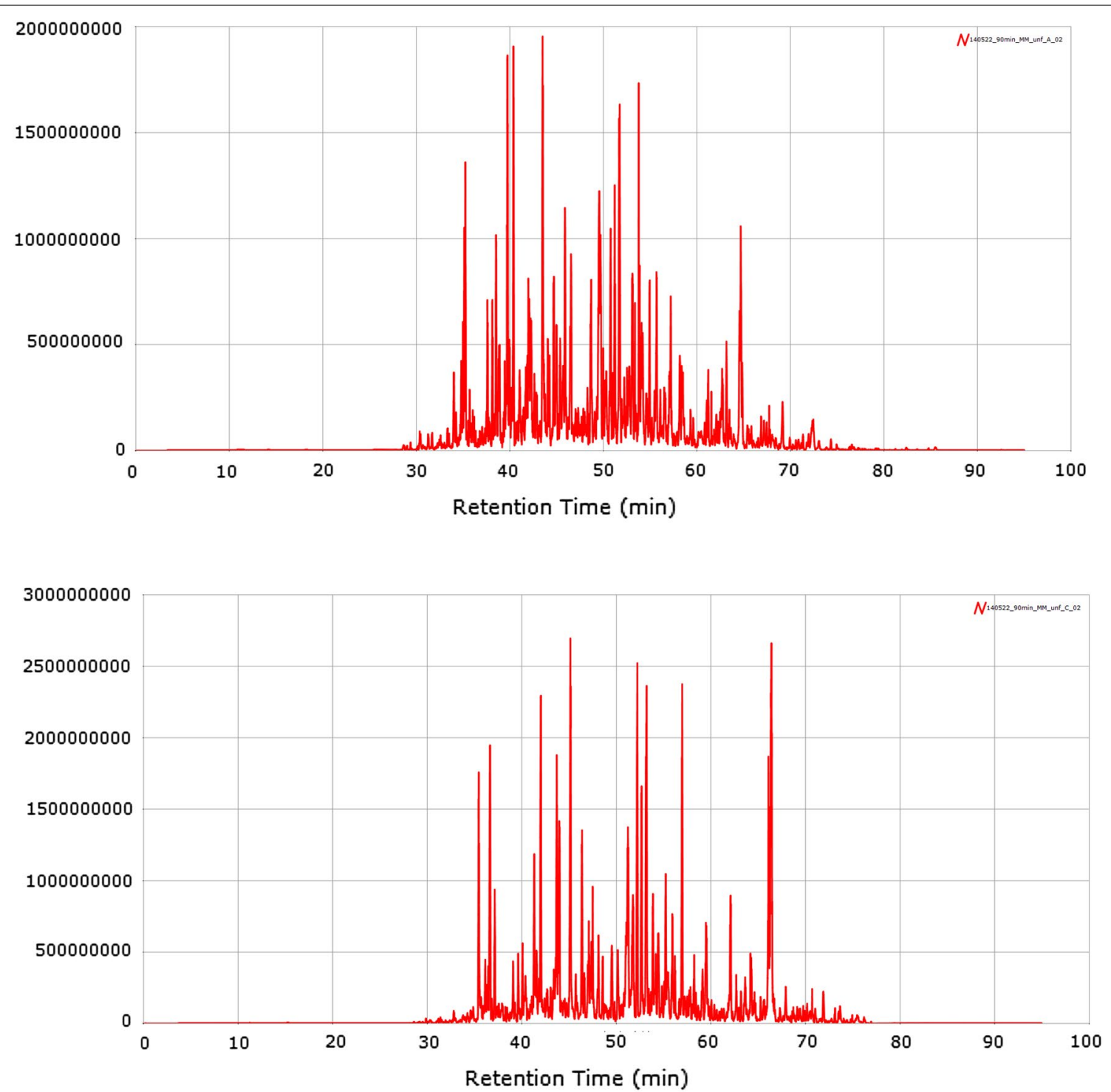

Fig 3 Resulting nano-chromatographic separation of biobank samples.

\section{Automated processing of $\mathbf{3 8 4}$ sample tubes}

Patient samples from the hospital were retrieved as outlined in Fig. 1, with the configured workflow steps being documented by barcode tagging. Frozen tissue sample processing was carried out as described in Fig. 2, where the solid tissue sections are homogenized, ending up as tissue extract solution samples. By this procedure, we are 
able to efficiently handle both biofluids and solubilized tissue extracts in the Sample Array Tube Handler system.

Automated sample processing is the only way to manage large sample numbers in sample archives where sample integrity is ensured $[15,16]$. The increase in the number of high quality biobank samples results in an increased demand for the most appropriate sample type from a given sample set for proteomics and/or genomic studies. Large sample collections also need detailed clinical records matched that are matched to the sample being readily available.

Correspondingly, in order for us to maintain the sample integrity and quality over time in large scale biobanking, we apply the principle of single usage [17]. Single usage means, by our definition, that for any given stored sample in a biobank archive, it will be thawed once and never re-entered into the biobank for further storage. By this principle, we will ensure that sample integrity of each and every biofluid fraction or tissue is not variably changed by repeated thawing and freezing cycles. This consideration is especially relevant to large cohort studies where samples can easily reach thousands in number, thus we need controlled, efficient and automated workflows $[18,19]$. The capacity of biobank sample collections is directly related to parallel automated processing. We are employing automated liquid handling instrumentations (HAMILTON robot MicroLab Starlet, Hamilton, Bonaduz AG, Switzerland) and high-density storage at $-80^{\circ} \mathrm{C}$ (LICONIC freezer STT1k5 ULT, Liconic AG, Mauren, Liechtenstein). Currently, our ultralow robotic temperature storage facilities $\left(-80^{\circ} \mathrm{C}\right)$, is able to store samples from clinical studies at a density of 5 million tubes using the 384-tube format, within a single freezer unit of approximately $3 \mathrm{~m}^{3}$ in volume.

In order to investigate the rigidity and stability of the Sample Array Tube Handler, we performed repeated picking and sorting steps in the 384 rack system. This was performed in an experimental setting using 4 different 384-racks filled with 384 separate sample tubes that were transferred over the platform board, from one end to the other.

The processing steps were performed according to the following protocol. A sample transfer scheme with specific XY positions was created in a method part of the Sample Array software. Tubes from the series of source plates (A) were then transferred to the destination series of plates (B) according to the designed scheme.

1. Open method part of the S384 software.

2. Design of the sample transfer with specific XY positions.

3. Pick up the sample tube from the A series of racks.
4. Deliver the sample tube from the A series of racks to the $B$ series of racks.

Each step within the method is monitored and a corresponding CSV file is generated. An example of the data output file generated after transfer is presented in Table 1, where the $\mathrm{X}-\mathrm{Y}$ coordinate of each sample tube transfered from A1, B1, C1 and D1 to the A2, B2, C2 and D2-384-tube plates are tracked.

The entire operation of $4 \times 384$ tubes is experimentally 1 cycle within our evaluation and validation of the S384 platform, integrated into a biobank environment. We have performed more than 100,000 single tube transfers on the platform with the protocol described above. The platform allows transfer of tubes from 10 source plates into 10 destination plates.

Each transfer step takes about $4.5 \mathrm{~s}$, which results in a total experimental time of close to $13 \mathrm{~h}$ non-stop processing for transfer of 10,000 tubes.

We have performed sample transfer experiments, both within a day, in-between days and in-between weeks. In total we have processed more than 100,000 transfers over a 1 month period. We had no failures reported throughout these experiments over a 1 month period.The data was generated at ambient temperature. We also repeated the same experiments at $-20^{\circ} \mathrm{C}$ and found the rigidity of the Sample Array platform to be very similar to that of the ambient performance. The resulting report file generated from each cycle with 10,000 sample tubes indicated no errors. Five cycles were studied.

The sample quality is maintained throughout the biobank handling processes which is illustrated in the resulting proteomics spectra shown in Fig. 3 where patient tissues were analysed by LC-MS using a $\mathrm{Q}$ Exactive platform.

\section{Sample tube distribution into multiple racks by scrambling} Large scale biobanks with millions of sample tubes need a given structure and organization in order to ensure the safety of the samples. In principle the sample archive should have a backup. In this respect, all sample tubes from a single patient should never be stored within one 384 rack. Instead, the sample tubes should be divided and spread into multiple plates. In addition, a backup of each sample collection, approx $10-20 \%$ should be stored physically in another center located elsewhere, in order to make sure that the samples will never be lost.

Scrambling of samples into multiple racks can easily be achieved with the Sample Array Tube Handler platform.

\section{Biobank space optimization and refilling the $\mathbf{3 8 4}$ racks}

With multiple studies going on at the same time the sample flow in and out of the biobank is highly active. The 


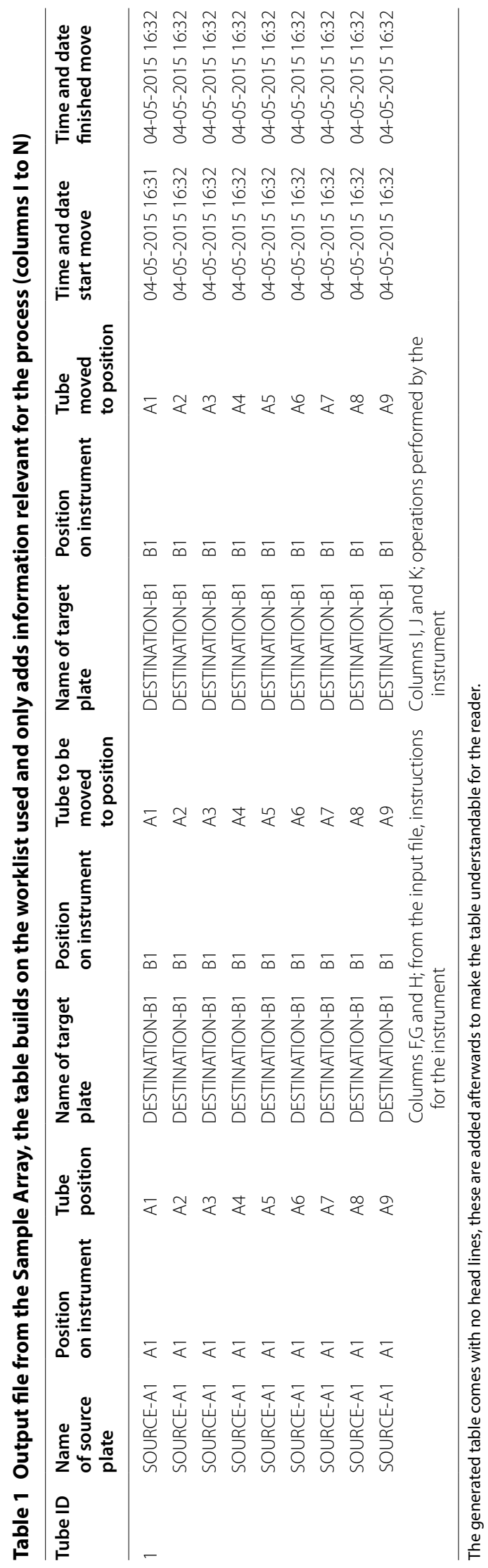


samples in a biobank will shift over time as samples are being collected and exported to research groups, in addition to the steady growth of the number of samples. These dynamic events will require that sample storage capacity is optimised.

Upon sample retrievals from biobanks, sample tubes from different 384-racks will be partly empty. In addition, the constant patient recruitment with tens of thousands of sample tubes per month makes it necessary to re-fill the 384-racks. The 384 robotic platform is an efficient work horse to fulfil this imoportant task.

\section{4-sample tube tightness}

The tightness of the tube sealing, when processed with the robotic fine mechanics arm, was investigated with tubes containing blood samples in liquid form $\left(23^{\circ} \mathrm{C}\right)$ as well as with frozen blood $\left(-20^{\circ} \mathrm{C}\right)$, and solubilized tissue extracts. The blood fractions from cancer and cardiovascular patient samples were processed as described in Fig. 1. The sample tubes were investigated by microscope in order to look for any deterioration in the alumina film that covers the 384 tubes. A sample set of 1,000 tubes was investigated at ambient temperature, and we could not see any traces of harm or deterioration on these tube coatings. Resistance to leakages was also investigated. The 84 racks were placed in a swing out centrifuge and centrifuged for $10 \mathrm{~min}$. Upon reinvestigation we examined the racks for any leakages caused by the centrifugation. None of the sample tubes containing plasma and erythrocyte blood fractions showed any trace of leakage, which was considered a confirmation of the excellent performance of the Sample Array Tube Handler.

\section{Laboratory information management system (LIMS)}

The data handling process workflow of our biobank infrastructure is outlined in Fig. 4, where the 384 robotic processing plays a central role in the overall handling of patient samples. Consequently, the CSV-file generated by the Sample Array Tube Handler platform for each of 384 plates is automatically fed into the database through the laboratory information management system (LIMS). The new positions of each and every tube are aligned within the given study in the LIMS. The 2D barcode makes it possible to track each tube and position within the 384 rack. The clinical data that is associated with the sample tube of each patient is also linked to the CSV-file from the robot.

\section{Workflow advantages}

The issue of poor biospecimen quality has often not been discovered until large number of clinical assays were performed or study reports already released [20].

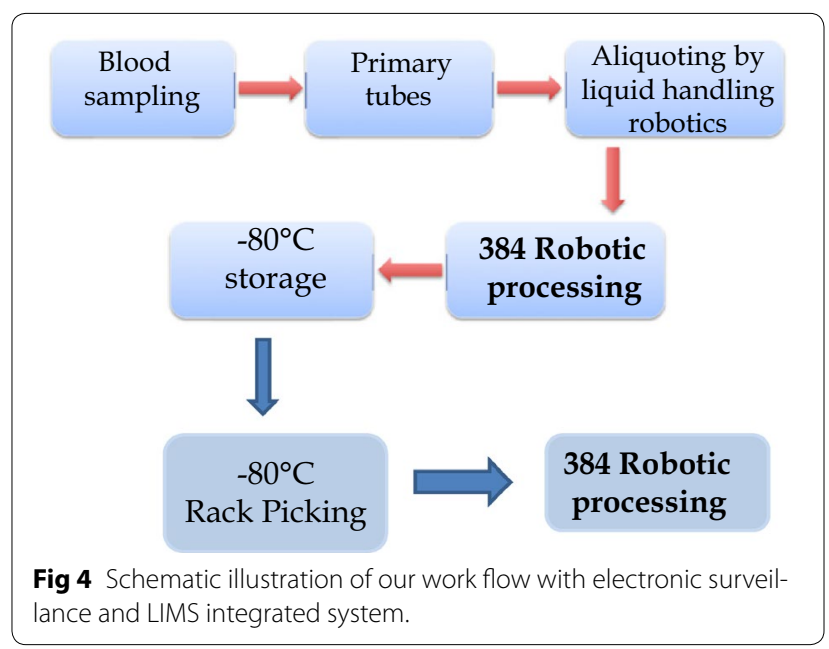

The present system generates $70 \mu \mathrm{L}$ aliquotes of whole blood (EDTA), plasma (EDTA, heparin, citrate), buffy coat (EDTA, heparin, citrate), red blood cells (EDTA, heparin, citrate) and serum in a 384-tube format using a fully automated sample processing strategy. This highly standardized procedure minimizes the probability of preanalytical errors and also makes possible the use of high quality samples on every occasion by avoiding the use of previously thawed samples.

The biobank practice can be implemented in clinical routine in hospital laboratories and has now gained acceptance within several health care units. Instead of larger 5-10 mL tubes or the 96-tube format the current system typically gives the biobank user access to hundreds of thousands of high quality small sample aliquots.

\section{Concluding remarks}

The impact of personalized medicine with targeted treatments is increasing and it is not surprising that developing personalized medicine methodologies now conquer much of the current development portfolio within big pharma and the biotech industry. Translating basic science to discover novel and improved treatments is a huge challenge [21, 22]. Automated picking and sorting of samples in biobanks and sample repositories is a strategically important part of modern healthcare operations in order to advance our understanding and knowledge of the molecular and environmental basis of human diseases. The ultimate healthcare goal that forms the basis of large recent investments in biobanks is to provide the prerequisites for refined and improved diagnostics and treatments. This will eventually aid in developing personalized medicine and the understanding of systems biology. 


\section{Authors' contributions}

Malm, Fehniger and Marko-Varga_overall responsibility and manuscript preparation. Lindberg, Steinfelder, Appelqvist, Yakovleva, Welinder—programming and robotic workflow. Erlinge - providing samples and clinical input. All authors read and approved the final manuscript.

\section{Author details}

1 Section for Clinical Chemistry, Department of Translational Medicine, Lund University, Skåne University Hospital Malmö, 20502 Malmö, Sweden. ${ }^{2}$ Centre of Excellence in Biological and Medical Mass Spectrometry, Biomedical Centre D13, Lund University, 22185 Lund, Sweden. ${ }^{3}$ Department of Biomedical Engineering, Clinical Protein Science and Imaging, Biomedical Center, Lund University, 22100 Lund, Sweden. ${ }^{4}$ Department of Cardiology, Lund University, Skåne University Hospital Lund, 22185 Lund, Sweden. ${ }^{5}$ ThermoFisher Scientific, Glasgow, UK. ${ }^{6}$ First Department of Surgery, Tokyo Medical University, 6-7-1 Nishishinjiku Shinjiku-ku, Tokyo 160-0023, Japan.

\section{Acknowledgements}

We would like to acknowledge Antal Banki for programing and robotic assistance. This work was supported by grants from the Swedish Academy of Pharmaceutical Sciences, C-HPP (HUPO), Swedish Research Council, the Swedish Foundation for Strategic Research (SSF, TOTAL AMI), Vinnova, Ingabritt \& Arne Lundbergs forskningsstiftelse, Fundacion Federico SA, and by the Crafoord Foundation.

\section{Compliance with ethical guidelines}

\section{Competing interests}

The author declares that they have no competing interest.

Received: 6 May 2015 Accepted: 2 July 2015

Published online: 14 August 2015

\section{References}

1. Riegman PHJ, Morente MM, Betsou F, De Blasio P, Geary P (2008) Marble Arch Int Working G Biobanking for better healthcare. Mol Oncol 2(3):213-222

2. Khleif SN, Doroshow JH, Hait WN (2010) AACR-FDA-NCl Cancer Biomarkers Collaborative Consensus Report: advancing the use of biomarkers in cancer drug development. Clin Cancer Res 16:3299-3318

3. Lasso RO (2010) The ethics of research biobanking. JAMA 304(8):908-910

4. Carraro P, Zago T, Plebani M (2012) Exploring the initial steps of the testing process: frequency and nature of preanalytic errors. Clin Chem 58:638-642

5. Ellervik C, Vaught J (2015) Preanalytical variables affecting the integrity of human biospecimens in biobanking. Clin Chem [Epub ahead of print]

6. Lippi G, Chance JJ, Church S, Dazzi P, Fontana R, Giavarina D et al (2011) Preanalytical quality improvement: from fram to reality. Clin Chem Lab Med 49(7):1113-1126
7. Schrol A-S, Würtz S, Kohn E, Banks RE, Nielsen HJ, Sweep FCGJ et al (2008) Banking of biological fluids for studies of disease-associated protein biomarkers. Mol Cell Prot. 7:2061-2066

8. Lane L, Bairoch A, Beavis RC, Deutsch EW, Gaudet P, Lundberg E et al (2014) Metrics for the Human Proteome Project 2013-2014 and strategies for finding missing proteins. J Proteome Res 13(1):15-20

9. Paik YK, Hancock WS (2012) Uniting ENCODE with genome-wide proteomics. Nature Biotech 30(11):1065-1067

10. Nilsson CL, Berven F, Selheim F, Liu H, Moskal JR, Kroes RA et al (2013) Chromosome 19 annotations with disease speciation: a first report from the global research consortium. J Proteome Res 12(1):135-150

11. Lichti CF, Liu H, Shavkunov AS, Mostovenko E, Sulman EP, Ezhilarasan R et al (2014) Integrated chromosome 19 transcriptomic and proteomic data sets derived from glioma cancer stem-cell lines. J Proteome Res 13(1):191-199

12. Marko-Varga G, Omenn GS, Paik YK, Hancock WS (2013) A first step toward completion of a genome-wide characterization of the human proteome. J Proteome Res 12:1-5

13. MacArthur DG, Balasubramanian S, Frankish A, Huang N, Morris J, Walter $K$ et al (2012) A systematic survey of loss-of-function variants in human protein-coding genes. Science 335(6070):823-828

14. Nilsson CL, Mostovenko E, Lichti CF, Ruggles K, Fenyö D, Rosenbloom KR et al (2015) Use of ENCODE resources to characterize novel proteoforms and missing proteins in the human proteome. J Proteome Res 14(2):603-608

15. Paik YK, Jeong SK, Omenn GS, Uhlen M, Hanash S, Cho SY et al (2012) The chromosome-centric human proteome project for cataloging proteins encoded in the genome. Nature Biotech 30(3):221-223

16. Marko-Varga G, Baker MS, Boja ES, Rodriguez H, Fehniger TE (2014) Biorepository regulatory frameworks: building parallel resources that both promote scientific investigation and protect human subjects. J Proteome Res 13(12):5319-5324

17. Malm J, Fehniger TE, Danmyr P, Végvári A, Welinder $C$, Lindberg $\mathrm{H}$ et al (2013) Biobanking work flow standardization — developments providing sample integrity. J Proteomics 95:38-45

18. Malm J, Danmyr P, Nilsson R, Appelqvist R, Végvári A, Marko-Varga G (2013) Blood sample standardization developments for large scale biobanking. J Proteome Res 12:3087-3092

19. Fehniger TE, Boja ES, Rodriguez H, Baker M, Marko-Varga G (2014) Four areas of engagement requiring strengthening in modern proteomics today. J Proteome Res 13(12):5310-5318

20. Vaught J, Lockhart N (2012) The evolution of biobanking best practices. Clin Chim Acta 413(19-20):1569-1575

21. Marko-Varga GA, Végvári Á, Fehniger TE (2011) A protein shake-up, Public service review. Eur Union 21:250-252

22. Végvári Á, Rezeli M, Döme B, Fehniger TE, Marko-Varga G (2011) Translation science for targeted personalized medicine treatments. In: Sanders $S$ (ed) Selected presentations from the 2011 Sino-American symposium on clinical and translational medicine. Science/AAAS, Washington DC, pp $36-37$

\section{Submit your manuscript to a SpringerOpen ${ }^{\odot}$ journal and benefit from:}

- Convenient online submission

- Rigorous peer review

- Immediate publication on acceptance

- Open access: articles freely available online

- High visibility within the field

- Retaining the copyright to your article

Submit your next manuscript at $>$ springeropen.com 